\title{
Artificial Neural Network Modeling of Flow Stress in Hot Rolling
}

\author{
Parya AGHASAFARI, ${ }^{1)}$ Hamid $\mathrm{ABDI}^{2)}$ and Mahmoud SALIMI ${ }^{1) *}$ \\ 1) Mechanical Engineering Department, Isfahan University of Technology, Isfahan, 8415683111 Iran. \\ 2) Center for Intelligent Systems Research, Deakin University, Waurn ponds 3216, Victoria, Australia.
}

(Received on September 30, 2013; accepted on December 9, 2013)

\begin{abstract}
In this study, an artificial neural network model is proposed to predict the flow stress variations during the hot rolling process. Optimization of the proposed neural network with respect to number of neurons within the hidden layer, different training methods and transfer functions of the neural network is performed. The results of the optimal network were compared with those of the conventional analytic method and it is shown that using an optimal neural network the mean calculated error is drastically reduced.
\end{abstract}

KEY WORDS: hot rolling; flow stress; artificial neural network; modeling; optimization.

\section{Introduction}

Hot rolling process is one of the most common processes in forming of metal products with different shape and dimensions. In this process, dimensional accuracy of the workpiece depends on accurate prediction of materials resistance to forming in addition to mill control system. Resistance against deformation mainly depends on surface contact of the workpiece and the rollers, flow stress and friction coefficient. Flow stress in rolling is influenced by various parameters such as grain size, recrystallization, dynamic and static recovery, dislocations distribution, reduction at each stand, temperature and velocity or strain rate. In sheet rolling industries, controlling the strip profile and flatness is an important issue. For this purpose the pressure applied on the work rolls neck to maintain the required force for the rolling process depends on the resistance against deformation. One way to estimate this force is by considering the chemical composition of material for modeling of the workpiece's mechanical behavior during the deformation. Extensive studies are conducted in this area including several works using Artificial Neural Networks (ANN) to model this process. Zhang et al., considered percentage of Carbon and Manganese content, input-output thickness, temperature and width of the plate as inputs of an ANN and tried to estimate the rolling force. ${ }^{1}$ Lin et al. modeled an ANN whose inputs were temperature, strain, strain rate, logarithm of deformation's rate and inter-pass delay time. Flow stress of two-pass hot deformed 42CrMo steel was regarded as network output. ${ }^{2)}$ In Ghaisari et al. model reduction, coiling temperature, finishing rolling temperature and percentage of components were inputs of ANN and outputs were the yield strength, ultimate tensile strength and elongation. ${ }^{3)}$ Bagheripoor and Bisadi regarded impressive parameters of

\footnotetext{
* Corresponding author: E-mail: salimi@cc.iut.ac.ir
}

DOI: http://dx.doi.org/10.2355/isijinternational.54.872 rolling process such as; thickness reduction, initial temperature, friction coefficient and roll speed as inputs of ANN. Calculated rolling forces and rolling torques from the finite element method were used as outputs of the ANN to monitor the dimension and plate output profile accuracy. ${ }^{4}$ Rao and Prasad tried to investigate the feasibility of utilizing an ANN to extract the complex relationships involved in hotdeformation process modeling. ${ }^{5)}$ From the production point of view it is very important to predict the required roll force as accurate as possible so that the desired outgoing thickness is maintained. The requirement for the large computational time using either accurate analytical solution or finite element method makes it unsuitable for online applications. In addition the most challenging parameter required for accurate prediction of the rolling force by any method is the workpiece resistance to deformation known as the flow stress. In present investigation, an attempt is made to obtain an optimized model for evaluating the flow stress in hot rolling process by using an inverse analysis method and ANN. The ANN results are compared with an analytical method based on the least square method of error between the calculated values and the measured ones.

\section{Flow Stress Equation}

The flow stress of material is generally dependent on parameters such as the composition $(C)$, temperature $(T)$, strain $(\varepsilon)$, strain rate $(\dot{\varepsilon})$, grain size $(d)$ and previous history. These parameters are not constant within the volume and also vary with time during the hot rolling process. The mechanisms by which the recrystallization and recovery take place are complicated and are not the purpose of this investigation. In this investigation the mean and equivalent values of the above mentioned parameters at each stand are considered to define the flow stress.

Researchers have presented different relations for flow stress. For instance, Ludwik ${ }^{6)}$ presented his model in terms 
of strain rate. Zener and Holman ${ }^{7,8)}$ and also Alder and Phillips ${ }^{9}$ expressed their model based on dependence of flow stress on strain and strain rate. Finally, Inouye and some other researchers expressed their model more complete than the early researchers. They considered dependence of flow stress on temperature, strain and strain rate. In flat strip rolling where the plate width remains constant, the plane strain condition applies. Considering a thickness reduction in plane strain flat rolling from $h_{0}$ to $h_{f}$, the thickness strain is defined as:

$$
d \varepsilon_{t}=\frac{d h}{h} \text { or } \varepsilon_{t}=\int_{h_{0}}^{h_{f}} d \varepsilon=\ln \frac{h_{f}}{h_{0}}
$$

In this relation $h$ is the instantaneous height of the element in the roll gap.

The effective strain in this condition would be:

$$
\varepsilon=\frac{2}{\sqrt{3}} \ln \frac{h_{f}}{h_{0}}
$$

The strain rate representing the deformation speed known as the rate of deformation tensor is easily derived from the Eulerian linear strain tensor as follows:

$$
\dot{\varepsilon}_{i j}=\frac{1}{2} \frac{d}{d t}\left(\frac{\partial u_{i}}{\partial x_{j}}+\frac{\partial u_{j}}{\partial x_{i}}\right)
$$

Where $u_{i}$ is the velocity vector at position $x_{i}$.

From the engineering point of view the strain rate is simply defined as follows:

$$
\dot{\varepsilon}=\frac{1}{h} \frac{d h}{d t}
$$

The mean strain rate used in this investigation is the one proposed by Ford and Alexander. ${ }^{10)}$

At any position in the roll gap the strain is defined as:

$$
\dot{\varepsilon}_{x}=\frac{1}{h} \frac{d h}{d x} \frac{d x}{d t}
$$

Where $x$ is the distance from the exit plane in the deformation zone and $h$ is the strip thickness at distance $x$. Since the term $\frac{d x}{d t}$ is the horizontal velocity of the element at distance $x$, by referring to geometry of the deformation zone and by integrating the strain over the arc of contact and dividing the result by the projected contact length, the mean strain rate for any pass is obtained as follows:

$$
\dot{\varepsilon}_{m}=\frac{\pi N}{30} \frac{\sqrt{R_{r} r}}{h_{0}} \frac{4-3 r}{(2-r)^{2}}
$$

In this relation $r$ is the reduction in thickness as given by $r=\frac{h_{f}-h_{0}}{h_{0}}$ and $N$ is the roll speed in RPM.

In this study the mean flow stress in the plastic zone is estimated using the mill data. Inouye's relation is used to describe the flow behavior of specific steel during hot rolling. The form of the equation is given by:

$$
\sigma=\sigma_{0} \varepsilon^{n} \dot{\varepsilon}^{m} \exp (A / T)
$$

Where $\sigma_{0}, n, m$ and $A$ are constants, $\mathcal{\varepsilon}, \dot{\varepsilon}$ and $\sigma$ represent the equivalent or effective strain, effective strain rate and flow stress values respectively and $T$ is the temperature.
The von Mises yield criterion gives:

$$
\sigma=\sqrt{\frac{2}{3}}\left(s_{i j} s_{i j}\right)^{1 / 2}
$$

Where $s_{i j}$ is the deviatoric part of the stress tensor.

Similarly the strain and strain rate values presented in this equation are the effective or the representative values. For example the effective plastic strain increment with respect to the strain increment tensor $d \varepsilon_{i j}$ is given by the following relation.

$$
d \varepsilon=\sqrt{\frac{2}{3}}\left(d \varepsilon_{i j} d \varepsilon_{i j}\right)^{1 / 2}
$$

To evaluate a relationship between the parameters an ANN is modeled to take strain, strain rate, temperature and a constant coefficient of unity related to $\sigma_{0}$ as its inputs and considered the flow stress as its output. The Bland and Ford model is utilized to calculate the flow stress. In this model the rolling condition is considered to be plane-strain, plane sections perpendicular to the direction of rolling remains plane, the friction coefficient is considered to be constant over the arc of contact and the elastic strains of the workpiece is assumed to be negligible. This model is presented for calculation of the roll force and torque as follow: ${ }^{10,11)}$

$$
\begin{gathered}
\left.P=w\left[\int_{0}^{\varphi_{n}} p^{+} R^{\prime} d \varphi+\int_{\varphi_{n}}^{\alpha} p^{-} R^{\prime} d \varphi\right]\right] . \\
\left.G=w \mu R^{\prime} R_{r}\left[\int_{\varphi_{n}}^{\alpha} p^{+} d \varphi-\int_{0}^{\varphi_{n}} p^{-} d \varphi\right]\right] .
\end{gathered}
$$

In these relations $w$ is the plate width $\mu$ is the friction coefficient modeled as coulomb model, $R_{r}$ is the roll radius, $R^{\prime}$ is the deformed roll radius (the value of which is usually calculated using Hitchcock ${ }^{12}$ approximate solution), $\varphi$ is the angular contact position, $\varphi_{\mathrm{n}}$ is the angular neutral position and $p^{+}$and $p^{-}$are the roll pressure at entry and exit side at the roll gap respectively as given by the following relations:

$$
\begin{gathered}
p^{+}=\frac{2 k h}{h_{f}}\left(1-\frac{t_{f}}{2 k}\right) e^{\mu H} . \\
p^{-}=\frac{2 k h}{h_{0}}\left(1-\frac{t_{b}}{2 k}\right) e^{\mu\left(H_{1}-H\right)}
\end{gathered}
$$

$k$ is the yield (flow) shear stress of material. According to von Mises yield criterion $k=\sigma / \sqrt{3}$ where $\sigma$ is the representative flow stress at each stand. $t_{f}$ and $t_{b}$ are the front and back tensions. $h, H$ and $H_{l}$ appeared in these equations are specified as below:

$$
\begin{aligned}
h & =h_{f}+2 R^{\prime}(1-\cos \varphi) \\
H & =2 \sqrt{\frac{R^{\prime}}{h_{f}}} \tan ^{-1}\left(\sqrt{\frac{R^{\prime}}{h_{f}} \phi}\right) . \\
H_{1} & =2 \sqrt{\frac{R^{\prime}}{h}} \tan ^{-1}\left(\sqrt{\frac{R^{\prime}}{h_{f}}} \alpha\right) .
\end{aligned}
$$

In these equations $h_{f}$ is the final thickness and $\alpha$ is the total contact angle.

Table 1 gives a sample of rolling data for specific steel 
Table 1. Data log of the rolled material with $\mathrm{w}=958 \mathrm{~mm}$.

\begin{tabular}{ccccccccccc}
\hline stands & $\begin{array}{c}\mathrm{h}_{0} \\
(\mathrm{~mm})\end{array}$ & \multicolumn{1}{c}{$\%$} & $\begin{array}{c}P \\
(\mathrm{kN})\end{array}$ & $\begin{array}{c}v_{R} \\
(\mathrm{~mm} / \mathrm{s})\end{array}$ & $\begin{array}{c}t_{f} \\
(\mathrm{kN} / \mathrm{mm} 2)\end{array}$ & $\begin{array}{c}t_{b} \\
(\mathrm{kN} / \mathrm{mm} 2)\end{array}$ & $\begin{array}{c}\varepsilon \\
(\operatorname{lnh} 1 / \mathrm{h} 2)\end{array}$ & $\begin{array}{c}\dot{\varepsilon} \\
(1 / \mathrm{s})\end{array}$ & $\begin{array}{c}\mathrm{T} \\
\left({ }^{\circ} \mathrm{K}\right)\end{array}$ & $\begin{array}{c}\mathrm{G} \\
(\mathrm{kN}-\mathrm{mm})\end{array}$ \\
\hline $\mathrm{F} 1$ & 35.625 & 31.833 & 11145 & 982 & 0.006 & 0 & 0.3832 & 5.9012 & 1239.3 & 339160 \\
$\mathrm{~F} 2$ & 24.284 & 44.318 & 13982 & 1709 & 0.0085 & 0.006 & 0.5855 & 15.8535 & 1229.1 & 345020 \\
$\mathrm{~F} 3$ & 13.522 & 36.61 & 13056 & 2702 & 0.0095 & 0.0085 & 0.4558 & 29.0877 & 1218.8 & 177390 \\
$\mathrm{~F} 4$ & 8.572 & 29.93 & 10581 & 3904 & 0.011 & 0.0095 & 0.3557 & 45.8602 & 1204 & 88033 \\
$\mathrm{~F} 5$ & 6.006 & 22.361 & 9248 & 5121 & 0.012 & 0.011 & 0.2531 & 59.4655 & 1186.5 & 51850 \\
$\mathrm{~F} 6$ & 4.663 & 18.6 & 8061 & 6347 & 0.014 & 0.012 & 0.2057 & 74.6887 & 1167.3 & 34288 \\
$\mathrm{~F} 7$ & 3.796 & 12.053 & 6297 & 7339 & 0 & 0.014 & 0.1286 & 74.4273 & 1146.1 & 25576 \\
\hline
\end{tabular}

Table 2. Calculated values for friction coefficient and flow stress.

\begin{tabular}{ccc}
\hline stands & $\mu$ & $\sigma$ \\
\hline F1 & 0.254 & 0.152486 \\
F2 & 0.257 & 0.173910 \\
F3 & 0.259 & 0.206636 \\
F4 & 0.259 & 0.210688 \\
F5 & 0.258 & 0.241464 \\
F6 & 0.259 & 0.248264 \\
F7 & 0.257 & 0.267512 \\
\hline
\end{tabular}

grade St37 from the finishing mill at Mobarakeh steel company. By solving nonlinear equations based on the Bland and Ford model, the flow stress and friction coefficient values are obtained as given in Table 2 .

The main object of this work is to investigate the flow stress variations in the finishing mill. Referring to the slip line field solution provided by Hill, ${ }^{13)}$ the mean pressure factor $\gamma_{m}$ within the roll gap in relation to the plastic zone geometry is given.

Considering the representative strain as given by the following relation

$$
\varepsilon=\frac{2}{\sqrt{3}} \gamma_{m} \varepsilon_{t}
$$

$\gamma_{m}$ can be regarded as a redundant work factor. The proportion of redundant work is equal to: $\left(\gamma_{m}-1\right) / \gamma_{m}$. In finishing mill however the ratio of thickness of strip to the contact length is less than unity, in this condition the pressure factor $\gamma_{m}=1$.

As $\gamma_{m}$ in all stands is considered to be unity, the redundant work is negligible and was ignored. Hence the stress values obtained in this work which is related to strain, strain rate and temperature is close to the true stress values of material within the roll gap.

\section{Artificial Neural Networks Model}

In the last two decades, different types of ANNs have been employed in various applications including NNs for input output mappings. The input output mapping networks are commonly used where the input-output relationship is unknown, complex, or difficult to obtain. This relationship is constructed in the NNs based on the network structure, network transfer functions, and tuning weight and bias parameters of the neurons of the network. A multi-layer Feed Forward ANN (FFANN) that is commonly used for such mapping consists of one input layer, one or more hidden layers, and one output layer. ${ }^{14)}$

\subsection{Methodology}

Implementing the methodology of ANN requires gathering and preparing sample data sets in the first stage and design of the network in the next stage. In the first step, it is important to note that the network can only be as accurate as the data because most of the networks are general and do not have any prior information about the data. Furthermore, FFANNs have shown good interpolation ability. However, the accuracy for extrapolating beyond the sample data range is weak.

The design stage normally consists of 1) pre-processing of the data and 2) designing of the network structure and 3) training network weights and biases parameters. Preprocessing includes eliminating wrong data to make sure that the data is correct and normalization of the input and target vectors. For the normalization process, a sample consist of input as $x_{k}(n)$ and output as $y_{l}(n)$ where $k$ and $l$ represent the input and output numbers respectively, and $n$ is the data sample for each input and output. The normalized values for the inputs and outputs are given by:

$$
\begin{aligned}
& \hat{x}_{k}(n)=\frac{x_{k}(n)-\bar{x}_{k}}{\max \left(x_{k}\right)-\min \left(x_{k}\right)} \\
& \hat{y}_{l}(n)=\frac{y_{l}(n)-\bar{y}_{l}}{\max \left(y_{l}\right)-\min \left(y_{l}\right)}
\end{aligned}
$$

where $\bar{x}_{k}=\frac{\sum_{n=1}^{N} x_{k}(n)}{N}, \bar{y}_{l}=\frac{\sum_{n=1}^{N} y_{l}(n)}{N}$ and $N$ is the total number of data.

For the design stage, FFANN with one hidden layer that is shown in Fig. 1 is used.

The proposed FFANN has initial structure of 4-N-1 consisting of three layers with 4 neurons at the input, $\mathrm{N}$ neurons in the hidden and one neuron at the output layer.

For the training stage, once the network structure and input are determined, then the weights and biases are randomly initialized and FFANN can be trained. During the training process, the data set of input and output were used to adjust the network parameters so that outputs for a given input are as close as possible to the desired output. Com- 
monly, during the training process the weights and biases of the network are iteratively adjusted to minimize the mean square error (mse) of the network. In this study the network has only one output and the mean square error would be:

$$
m s e=\frac{1}{K} \sum_{n=1}^{K}\left[t_{l}(n)-a_{l}(n)\right]^{2}
$$

$t_{l}(n)$ is a vector of desired values, and $a_{l}(n)$ is the vector of the network's output values and $K$ is the number of training data.

The correlation coefficient $R^{14)}$ is often used to qualify the generalization capability of the training, validation and testing network. Correlation coefficient $R$ for the two variables is defined as the covariance of the two variables divided by the product of their standard deviations. One set of variables in this investigation is considered as the experimental flow stress obtained from the mill data using the Bland and Ford model and the other set of variables is referred to the flow stress predicted by the Neural Networks. In this case the expression for the correlation coefficient is defined as follows:

$$
R=\frac{\sum_{i=1}^{N}\left(\sigma_{e i}-\bar{\sigma}_{e}\right)\left(\sigma_{N i}-\bar{\sigma}_{N}\right)}{\sqrt{\sum_{i=1}^{N}\left(\sigma_{e i}-\bar{\sigma}_{e}\right)^{2} \sum_{i=1}^{N}\left(\sigma_{N i}-\bar{\sigma}_{N}\right)^{2}}}
$$

Different training algorithms are used for FFANN and they commonly use the gradient of the mean square error function to determine how to adjust the weights. Gradient descent and Gradient descent with momentum are two of the training algorithms for FFANN, however, they are often too slow for practical problems. Some of the other training algorithms are: Variable learning rate gradient descent, Bayesian regularization, Conjugate gradient with Powell/Beale restarts, Fletcher-Powell conjugate gradient, Polak-Ribiére conjugate gradient, One step scan, Scaled conjugate gradient, BFGS Quasi-Newton and Levenberg-Marquardt algorithm.

For the training purpose, the data is divided into three subsets of the "Training set", which is used for network training, the "Validation set", which is used to monitor the error during the training process and the "Test set" which was not already used during the training, but it is used to compare different models.

\section{Prediction of Flow Stress by FFANN}

In this paper, the aim was to obtain an ANN model for flow stress in hot rolling process.

As it was mentioned in a hot rolling process, flow stress

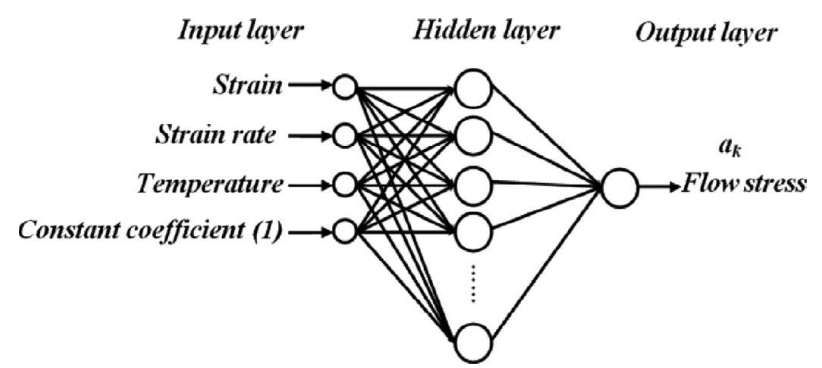

Fig. 1. The proposed FFANN. is a function of an initial stress $\left(\sigma_{0}\right)$, strain, strain rate and temperature. These parameters are considered as the inputs to a FFANN and the flow stress will be the output of the network. The aim is to obtain an optimal structure, network functions and parameters for a FFANN for this problem. In order to obtain such network, the following studies were made.

a-Different training algorithms for the FFANN with different transfer function and number of neurons in the hidden layer were used and their influences on rolling force accuracy were investigated.

b-Hidden layer neurons number for the best training algorithm and transfer function was changed and its influences on rolling force accuracy were obtained.

c-The optimal FFANN model implemented and trained with the best training algorithm, transfer function and number of neurons in the hidden layer.

\subsection{Methodology of Optimal FFANN for Flow Stress Prediction}

In this study, a MATLAB code for a $4-\mathrm{N}-1$ FFANN is developed. 100 datasets information for each rolling stand in finishing rolling mill were collected from the Mobarakeh Steel Company and used to design the network. The nonlinear equations based on Bland and Ford model were solved first and the flow stress as well as the friction coefficient were obtained. The aim is to obtain an optimal network that could be used to estimate the flow stress in terms of $\sigma_{0}, T$, $\varepsilon$ and $\dot{\varepsilon}$. An initial model (4-N-1) for FFANN with $(1, T, \varepsilon$, $\dot{\varepsilon})$ as its inputs and $\sigma$ as output was considered. To obtain the optimal structure of the FFANN, the training algorithm, hidden layer transfer function and neurons number were changed and the output of the network for each input vector was extracted. Rolling force for each network output based on Bland and Ford model was recalculated and the mean error values for all calculations were compared. Optimal structure for the FFANN based on the minimum of mean error values in rolling force recalculation was obtained. The flowchart of the method used is given in Fig. 2.

As it mentioned there are different training algorithm for FFANN. Also, different transfer functions are used in network layers. In this study the effects of different transfer functions for the hidden layer and different training algorithm are examined and the results are presented as given in Table 3.

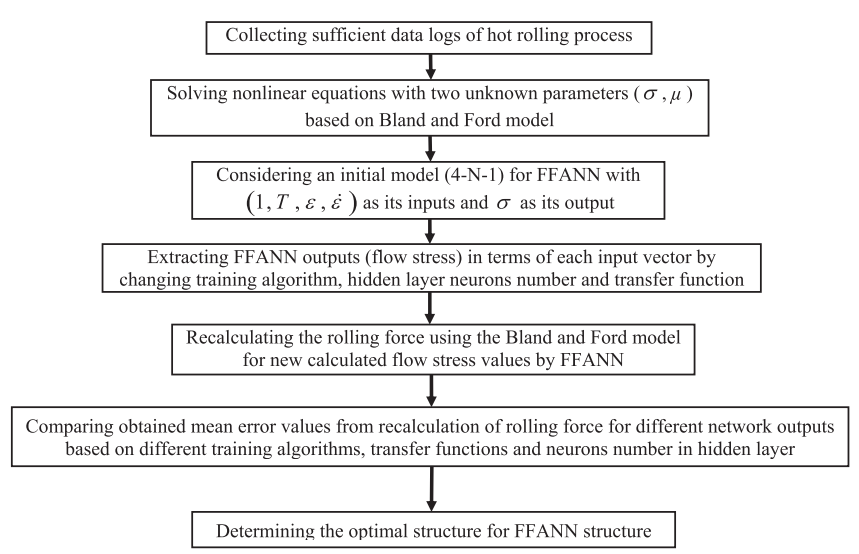

Fig. 2. Methodology flow chart. 
Table 3. Effects of different training algorithms and transfer functions on minimum of mean error in rolling force recalculation.

\begin{tabular}{lccc}
\hline \multirow{2}{*}{\multicolumn{1}{c}{ Training algorithm }} & \multicolumn{3}{c}{ Minimum of mean error } \\
\cline { 2 - 4 } & tansigmoid & logsigmoid & Linear \\
\hline Levenberg-Marquardt & 3.379331 & 3.643428 & 4.946779 \\
BFGS Quasi-Newton & 4.069595 & 4.024929 & 4.965345 \\
Resilient Backpropagation & 4.07842 & 4.213665 & 4.97255 \\
Scaled Conjugate Gradient & 4.284905 & 4.367531 & 4.974842 \\
Conjugate Gradient with Powell/Beale Restarts & 4.04859 & 4.370926 & 4.977647 \\
Fletcher-Powell Conjugate Gradient & 4.150404 & 4.103811 & 4.992811 \\
Polak-Ribiére Conjugate Gradient & 4.288098 & 4.427172 & 4.961029 \\
One Step Secant & 4.309429 & 4.394917 & 4.954902 \\
Variable Learning Rate Gradient Descent & 5.206061 & 5.309833 & 4.980747 \\
\hline
\end{tabular}

As it can be seen Levenberg-Marquardt algorithm and tangential sigmoid function give the lowest error values in rolling force recalculation. Here nine common training algorithms and three common transfer functions are considered and their effects on output accuracy were investigated.

Among the mentioned algorithms, the LevenbergMarquard (LM) algorithm is widely accepted as the most efficient one in the logic of recognition accuracy. The LM algorithm provides a numerical solution to the problem of minimizing a non-linear function. It is fast and has stable convergence. LM algorithm is basically a Hessian-based algorithm for nonlinear least square optimization. Hessianbased algorithms allow the network to learn more fine features of a complicated mapping. The training process converges quickly as the solution is approached, since the Hessian does not vanish at the solution. Many other methods have already been developed for ANNs training. The steepest descent algorithm is one of them which could be regarded as one of the most significant breakthroughs for training ANNs. LM can be thought of as a combination of steepest descent and the Gauss- Newton method. When the current solution is close to a local minimum it becomes GaussNewton method and show fast convergence while the solution is far from local minimum the algorithm behave like a steepest decent method. In the ANNs field, this algorithm is suitable for training problems. The detailed analysis of the LM algorithm is beyond the scope of this paper and interested readers are referred to the paper by Hagan and Mahnaj ${ }^{15)}$

The choice of transfer functions may strongly influence the performance of ANNs. The ANNs commonly use the sigmoid transfer functions. Sigmoid functions all share a similar S shape that is essentially linear in their center and nonlinear toward their boundaries. The log-sigmoid transfer function only returns positive values. If the ANN needs to return negative numbers, this function will be unsuitable. While tan-sigmoid transfer functions is positive and negative compatible version of the sigmoid function. The sigmoid transfer functions usually are the default choices for the FFANNs. However, it cannot be said for sure such functions should always provide optimal decision margins.

In this study the model performance is evaluated only in terms of its output accuracy and not considering the CPU
Table 4. Effects of hidden layer neurons number on minimum of mean error in rolling force recalculation.

\begin{tabular}{cc}
\hline neurons number & Minimum of mean error \\
\hline 1 & 4.871778743 \\
2 & 3.968010282 \\
3 & 4.858454337 \\
4 & 4.05080325 \\
5 & 4.309615419 \\
6 & 4.035406753 \\
7 & 4.036083442 \\
8 & 4.084914614 \\
9 & 3.871305441 \\
10 & 3.379330672 \\
11 & 4.02898009 \\
12 & 3.793245776 \\
13 & 4.174313535 \\
14 & 4.01370755 \\
\hline
\end{tabular}

time. As expected the combination of LM as the more suitable algorithm in training and the tan-sigmoidal transfer function as the most common and fitting transfer function presented the best results.

In next step, effects of different hidden layer neurons number for the best training algorithm and function is investigated. For this purpose, for the 4-N-1 networks the number $\mathrm{N}$ is altered from 1 to 14 as presented in Table 4.

It can be seen that 10 neurons numbers gives the best results among the others. Hence, 4-10-1 is chosen to be the best configuration for the FFANN.

The comparisons between the desired flow stress values obtained from the Bland and Ford model and the network output values for the training, validating and testing datasets are implemented.

In Fig. 3, the scatter plots and correlation coefficient for (a) training, (b) validating, (c) testing and (d) overall "training + validating + testing" of the network results are given. For a linear correlation or regression, straight lines of best fit (the trend-lines) which specify the correlation 


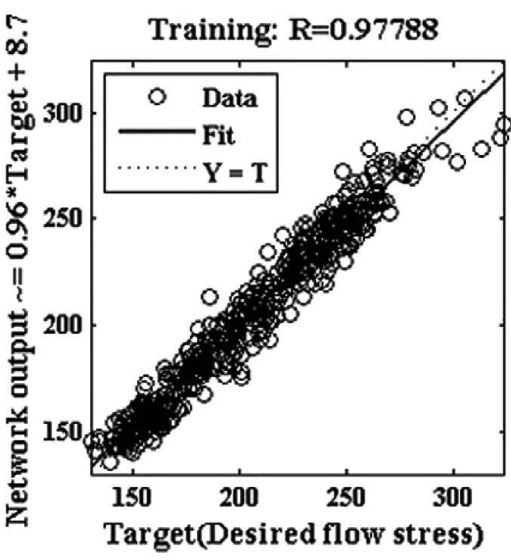

(a)

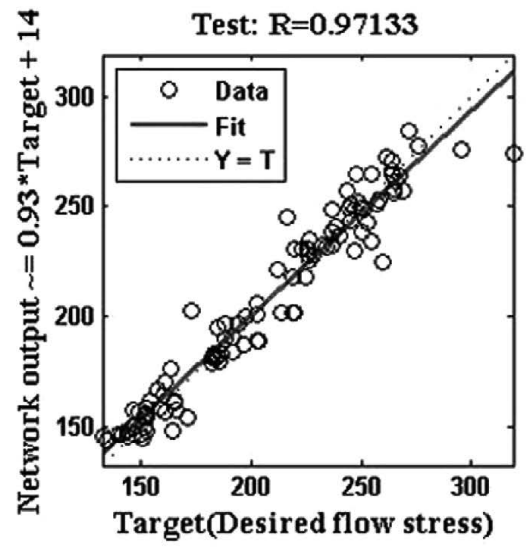

(c)

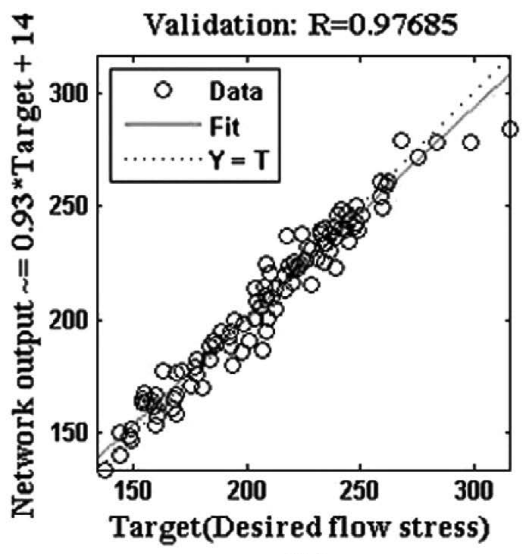

(b)

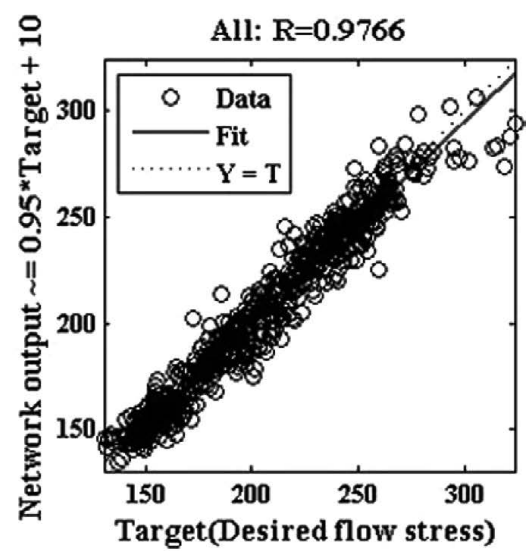

(d)

Fig. 3. Scatter plots and correlation coefficient for training (a), validating (b), testing (c) and overall (d) of the network results.

between the variables are presented on the vertical axis. The more the two data sets agree, the more the scatters tend to get closer to the identity line $(y=x)$. Since the values of correlation coefficient " $\mathrm{R}$ " in all plots is well above 0.9 and close to unity, indicates a strong positive relationship through a compact linear rule.

As it can be seen in Fig. 3(d) in which all values (training, validating and testing) are correlated by $\mathrm{R}=0.9766$, indicates close agreement between these values. It seems that satisfactory correlation between desired values and network output suggests that the FFANN is able to successfully predict the flow stress for the specific steel grade St37 from the finishing mill at Mobarakeh steel company.

\section{Comparison between the Results of ANN and the Analytical Method}

In Figs. 4(a) and 4(b) the results obtained from the FFANN and an analytical method based on a least-square logic is compared.

As it can be seen the FFANN result presents a more accurate prediction for the rolling force. Referring to the result of the analytical method, it can be seen that the percentage error of stands number 1, 5, 6 and 7 are generally lower and the rest are generally higher than the mean error. FFANN gives even distributed variations in percentage error in addition to more accurate results.

\section{Sensitivity Analysis}

Mathematically sensitivity is defined as the ratio of the reaction or change induced in the output to a stimulus or change in the input. Sensitivity analysis is used to evaluate the influence of model parameters on model predictions. This will help to study a model from different points of view such as: better understanding of the relationships between input and output variables, testing the robustness of the results of a model etc.

There are two methods of sensitivity analysis; local method and global method. In local method variables or parameters are varied one at a time by a small amount around fixed points and the effect on the output is calculated. In global method all variables or parameters are varied simultaneously over their entire feasible space and the effects on the output of individual variables are evaluated. In this study the global method is applied. As the ANN models are fundamentally nonlinear, the sensitivity measurement depends on the input values.

In this study which is related to hot rolling finishing mill, the four parameters or inputs (initial stress, strain, strain rate and temperature) are somewhat independent of each other. Initial stress is related to the material being rolled, strain is related to the roll gap adjustment which is controlled by the mill control unit or manually by an operator, strain rate is directly related to the rolling speed and the roll gap setting controlled by mill control unit and finally the material tem- 


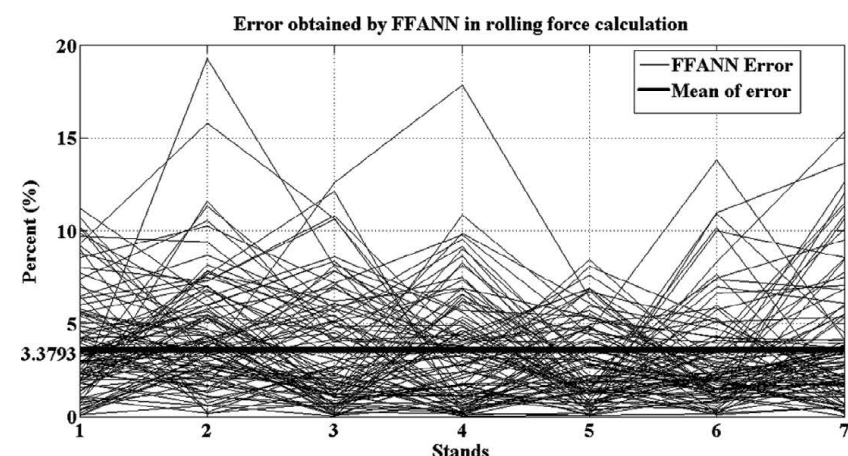

(a)

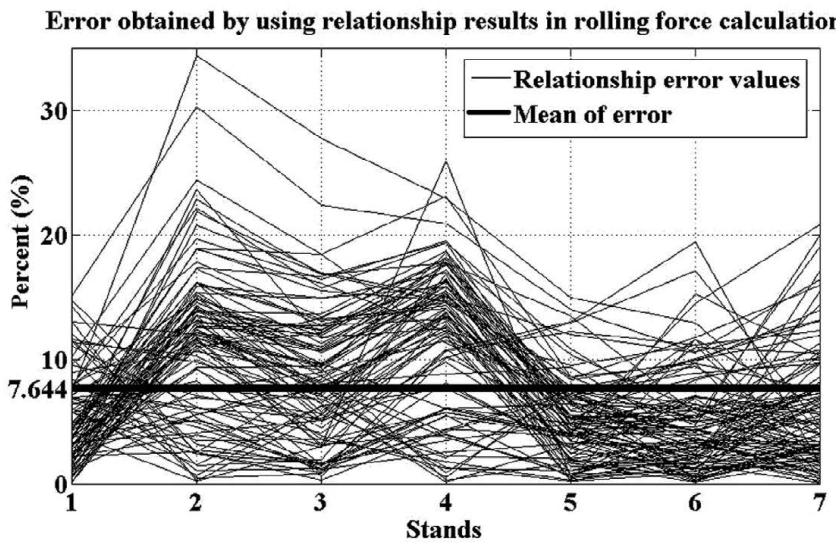

(b)

Fig. 4. Comparison between analytical results and NN results. a) $\mathrm{NN}$ results. b) analytical results.

perature which is related to heat transfer to rolls and environments (only a small fraction of the heat generated is due to the dissipated plastic work related to strain/strain rate). These four parameters directly affect the flow stress which in turn determines the rolling force and rolling torque required for the process. Hence, in this study for a particular material being rolled, the correlation between the value of flow stress and the three values (strain, strain rate and temperature) is provided.

To investigate the sensitivity of mechanical behavior of special steel grade St37 to different input parameters, graphs of flow stress in terms of each input are plotted. Using data from various datasets of finishing mill provides a wide range of variation in each input parameter.

For all 700 datasets where in early stands the strain and temperature are higher and the strain rate is lower, while in last stands where the strain is lower and the temperature at the end will drop by almost $20 \%$ and the work-piece experiencing higher strain rates the outputs are extracted. The scatter plots of flow stress against each variable are obtained and the best curve among the points is sketched by curve fitting. Figures 5, 6 and 7 show that all variables influencing the output and demonstrate suitable sensitivity of flow stress to each variable.

Since, in finishing mill data logs, high strains are related to early stands and in these stands the workpiece has higher temperatures, in these stands it shows a lower resistance against deformation, as shown in Fig. 5. In Fig. 6 it can be seen that in hot rolling process at low strain rate which is related to early stands at high temperature, material resis-

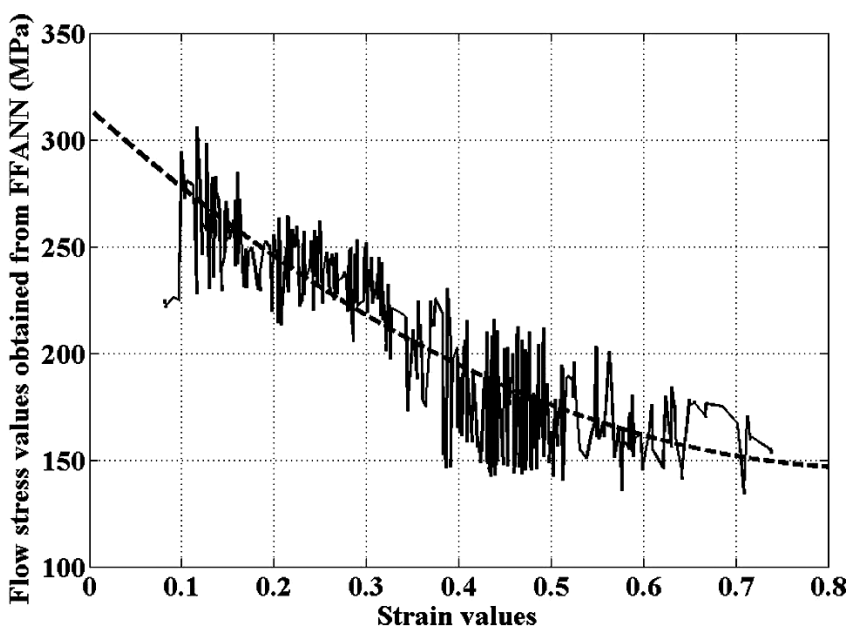

Fig. 5. Sensitivity of flow stress to strain.

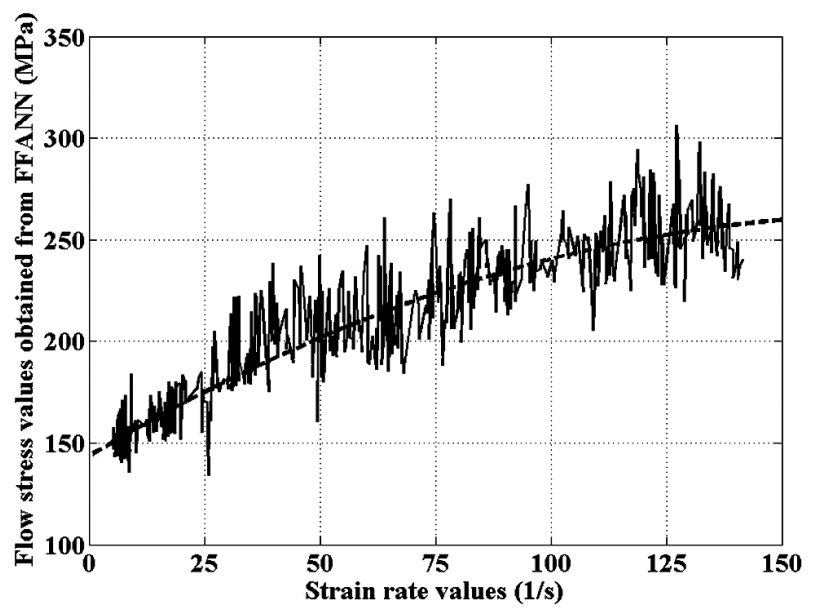

Fig. 6. Sensitivity of flow stress to strain rate.

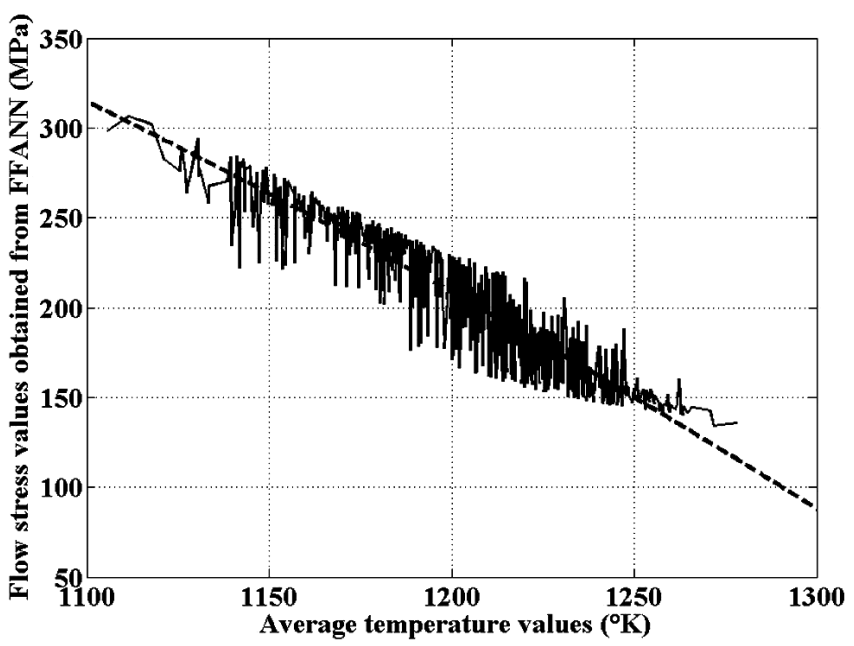

Fig. 7. Sensitivity of flow stress to average temperature.

tance against deformation is less than that of the other stands. In Fig. 7 sensitivity of flow stress to average temperature is given. It is obvious that in early stands where the temperatures are higher, the deformation takes place with lower stresses than the last stands. Thus, according to rolling mechanism all above figures show convenient sensitivity of flow stress to ANN inputs. 


\section{Conclusion}

In this study, an ANN model is proposed that was able to predict the flow stress variations during hot rolling process. The proposed network is a FFANN with one hidden layer and was optimized with respect to number of neurons within the hidden layer, different training Algorithms and transfer functions of the ANN. The result of the optimal network were compared with that of the conventional analytic method and it is shown that by employing an optimal FFANN the mean calculated error is reduced by about $50 \%$. It is concluded that the proposed method provides accurate result with reduced computational time and is a proper method for online control or optimization.

\section{List of symbols}

$p$ roll force

$G$ torque per roll

$R$ correlation coefficient

$R_{r}$ rollers radius

$T$ average temperature at each stand

$h_{f}$ Outgoing strip thickness

$h_{0} \quad$ Ingoing strip thickness

$k \quad$ Yield shear stress

$m$ sensitivity of flow stress to strain rate

$n$ sensitivity of flow stress to strain

$p^{+}$roll pressures at exit zone

$p^{-}$roll pressures at entry zone

$t_{b}$ backward tension

$t_{f}$ forward tension

$v_{R} \quad$ Roll surface speed

w Strip width $\alpha$ Total contact angle

$\varepsilon \quad$ effective strain

$\dot{\varepsilon} \quad$ effective strain rate

$\varphi$ contact angle position

$\varphi_{n}$ contact angle at neutral point

$\mu$ friction factor

$\sigma$ flow stress

$\sigma_{e}$ experimental flow stress values

$\bar{\sigma}_{e}$ Mean experimental flow stress

$\sigma_{N}$ flow stress values predicted by NN

$\bar{\sigma}_{N}$ mean values of $\sigma_{N}$

\section{REFERENCES}

1) L. Zhang, L. Y. Zhang, J. Wang and F. Ma: J. Iron Steel Res. Int., 14 (2007), 42.

2) Y. C. Lin, G. Liu, M.-S. Chen and J. Zhong: J. Mater. Process Technol., 209 (2009), 4611.

3) J. Ghaisari, H. Jannesari and M. Vatani: Adv. Eng. Softw., 45 (2012), 91.

4) M. Bagheripoor and H. Bisadi: Appl. Math. Model. Int., 37 (2013), 4593.

5) K. P. Rao and Y. K. D. V. Prasad: J. Mater. Process. Technol., 53 (1995), 552.

6) P. Ludwik: Elemente der Technologischen Mechanik, Springer-Verlag OHG, Berlin, (1909), 32

7) C. Zener and J. H. Hollomon: J. Appl. Phys., 17 (1946), 69.

8) J. T. Staley and J. Campbell: J. Matter. Sci. Lett., 19 (2000), 2179.

9) J. F. Alder and V. A. Phillips: J. Inst. Met., 83 (1954), 80.

10) H. Ford and J. M. Alexander: J. Inst. Mech., 92 (1964), 397.

11) D. R. Bland and H. Ford: Proc. Inst. Mech. Engrs., 159 (1948), 144.

12) J. Hitchcock: Roll Neck Bearing, Appendix I, Am. Soc. Mech. Eng, New York, (1935), 286.

13) R. Hill: Mathematical Theory of Plasticity, Oxford University Press, London, (1950), 245.

14) M. T. Hagan, H. B. Demuth and M. H. Beale: Neural Network Design, Colorado University Campus Publishing Service, (2002), 130.

15) M. T. Hagan and M. B. Mehnaj: IEEE Trans. on Neural Networks, 5 (1994), No. 6. 